\title{
A conjectured formula for Fully Packed Loop configurations in a triangle
}

\author{
Paul Zinn-Justin ${ }^{*}$ \\ LPTMS (CNRS, UMR 8626), Univ Paris-Sud \\ 91405 Orsay Cedex, France \\ and \\ LPTHE (CNRS, UMR 7589), Univ Pierre et Marie Curie-Paris 6 \\ 75252 Paris Cedex, France, \\ pzinn @lpthe.jussieu.fr \\ Submitted: Jan 15, 2010; Accepted: Jul 30, 2010; Published: Aug 9, 2010 \\ Mathematics Subject Classification: 05A15
}

\begin{abstract}
We describe a new conjecture involving Fully Packed Loop counting which relates (via the Razumov-Stroganov conjecture) recent observations of Thapper to formulae in the Temperley-Lieb model of loops.
\end{abstract}

*PZJ was supported by EU Marie Curie Research Training Networks "ENRAGE" MRTN-CT-2004005616, "ENIGMA" MRT-CT-2004-5652, ESF program "MISGAM" and ANR program "GIMP" ANR05-BLAN-0029-01.

${ }^{\dagger}$ The author wants to thank R. Langer for her participation in the early stages of this project, J. Thapper for sharing his numerical data, as well as P. Di Francesco, T. Fonseca and J.-B. Zuber for discussions. 


\section{Contents}

1 Introduction $\quad 3$

2 Preliminaries 3

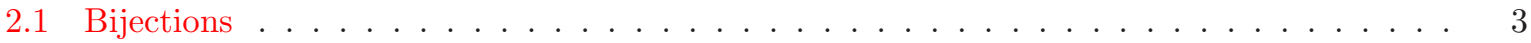

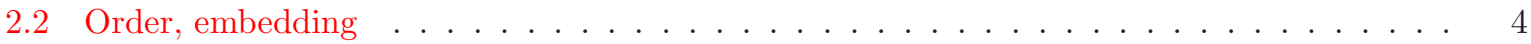

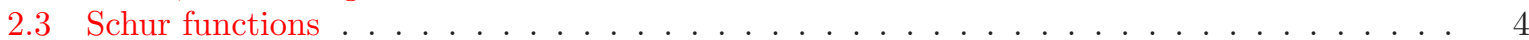

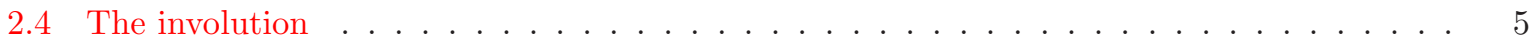

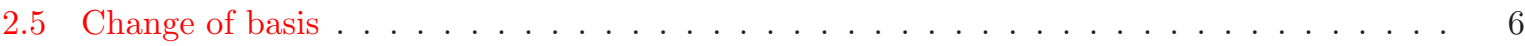

3 Statistical models of loops and Razumov-Stroganov conjecture $\quad 7$

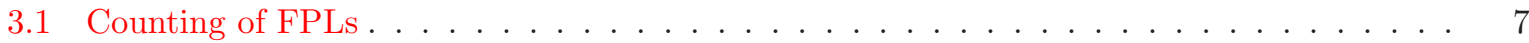

3.2 The Temperley-Lieb(1) loop model . . . . . . . . . . . . . . . . . . . . . . . 9

3.3 The Razumov-Stroganov conjecture . . . . . . . . . . . . . . . . . . . . 10

4 The formula $\quad 10$

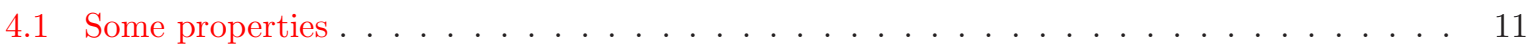

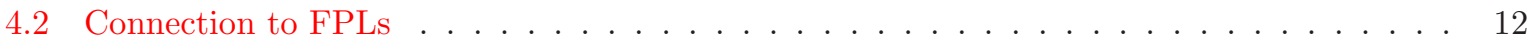

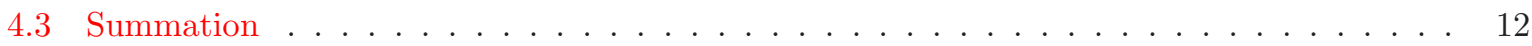

4.4 Special cases . . . . . . . . . . . . . . . . . . . . 13

5 Analogues of Thapper's conjectures $\quad 14$

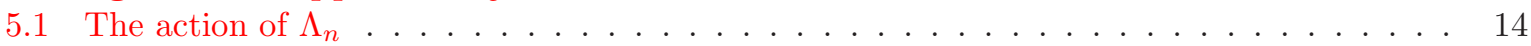

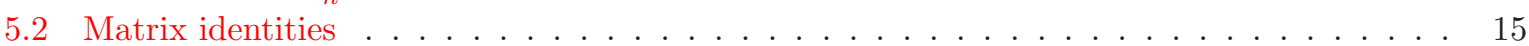

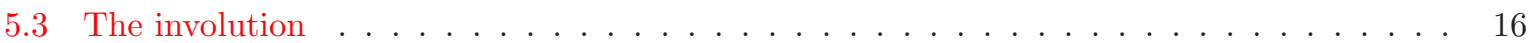

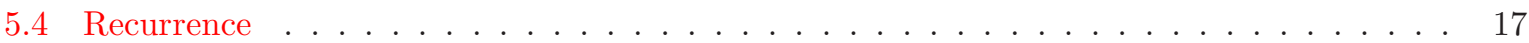

5.5 Largest component . . . . . . . . . . . . . . . . . . . . . . 18

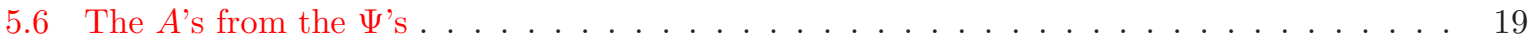

6 The $\tau$-generalization $\quad 19$

$\begin{array}{ll}\text { A Existence of the matrix } K & 21\end{array}$

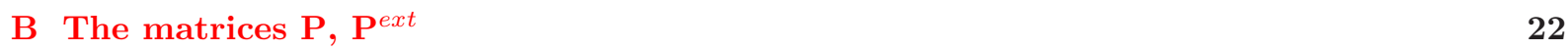

C Example of ground state entry of the Temperley-Lieb loop model 22

D Example of matrices $\bar{A}$ and C $\quad 23$ 


\section{Introduction}

In the literature generated by the seminal papers [1, 19] and revolving around the so-called Razumov-Stroganov (RS) conjecture, it has often been remarked that there are more conjectures than theorems. The present work, sadly, will not help correct this imbalance: it is entirely based on one more conjecture. The latter, however, is of some interest since it connects the two sides of the Razumov-Stroganov conjecture; that is, it expresses the number of Fully Packed Loop configurations (FPLs) in a triangle with certain boundary conditions as the constant term of a quasi-generating function which is closely related to expressions appearing in the context of the Temperley-Lieb(1) (sometimes called $O(1)$ ) model of loops [10, 20,9]. This formula was inspired by an attempt to understand the observations of Thapper [22] on the enumeration of FPLs with prescribed connectivity, itself based on earlier work [3, 4]. In fact, we shall show in what follows that our new conjecture implies both the RS conjecture [19] and the conjectures of [22]. Since this article was written, the RS conjecture was proved in [2]; however there is no direct connection between our results and this proof, which provides no explicit formulae for the counting of FPLs.

The paper is organized as follows. The next section contains some basic definitions. In Section 3, we briefly recall the various statistical models involved and the required content from the work referred to above. Section 4 contains the main formula of this paper, its conjectural meaning, and the connection to the Razumov-Stroganov conjecture. Section 5 provides the link to Thapper's conjectures. The final section briefly describes the introduction of an extra parameter $\boldsymbol{\tau}$ in the formulae. Technical details and numerical results are to be found in the appendices.

\section{Preliminaries}

\subsection{Bijections}

Let $n$ be a positive integer. Various sets are in bijection:

1. the set of Ferrers diagrams contained inside the "staircase" Ferrers diagram (denoted by $\Gamma_{n}$ in what follows) with rows of lengths $n-1, n-2, \ldots, 1$;

2. the set of Dyck paths of length $2 n$;

3. the set of link patterns of size $2 n$, that is planar pairings of $2 n$ points inside a half-plane (the points sitting on the boundary);

4. the set of sequences of integers $\left(\alpha_{i}\right)_{i=0, \ldots, n-1}$ such that $\alpha_{i+1}>\alpha_{i}$ and $0 \leqslant \alpha_{i} \leqslant 2 i$ for all $i$.

These various bijections are described in Fig. 1. The only bijection which we shall write down explicitly is from Ferrers diagrams to increasing sequences: starting from a diagram $\alpha \subset \Gamma_{n}$, consider the sequence of lengths of its rows and pad it with zeroes so that it 

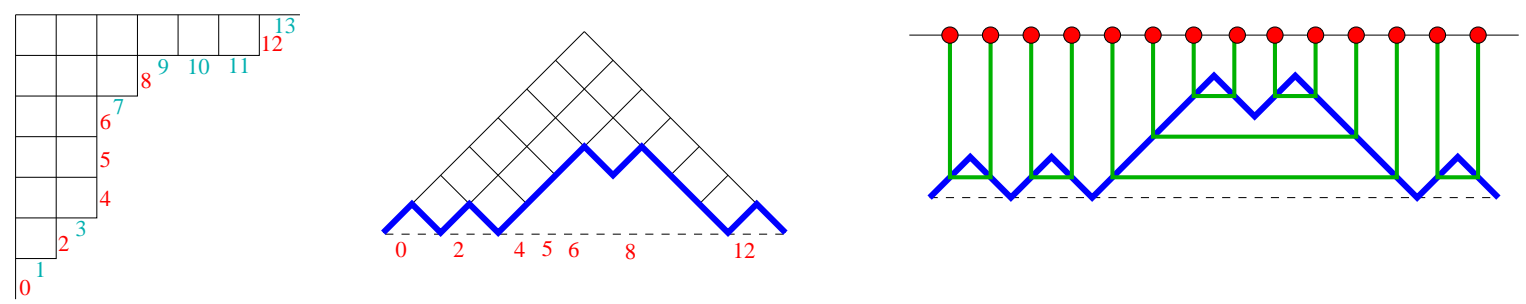

Figure 1: Bijections. From left to right: Ferrers diagrams and sequences of increasing integers; Ferrers diagrams and Dyck paths; Dyck paths and link patterns.

has exactly $n$ parts $\tilde{\alpha}_{1}, \ldots, \tilde{\alpha}_{n}$ (with in particular $\tilde{\alpha}_{n}=0$ ); the sequence is then given by $\alpha_{i}=\tilde{\alpha}_{n-i}+i, i=0, \ldots, n-1$.

We shall mostly use in what follows Ferrers diagrams and increasing sequences, identified via the bijection above. We shall call $\mathbf{A}_{n}$ their set.

\subsection{Order, embedding}

We call $|\alpha|$ the number of boxes of $\alpha \in \mathbf{A}_{n}:|\alpha|=\sum_{i=0}^{n-1}\left(\alpha_{i}-i\right)$.

We consider the partial order on Ferrers diagrams which is simply inclusion. In terms of sequences, $\alpha \subset \beta$ iff $\alpha_{i} \leqslant \beta_{i}$ for all $i$. The smallest element is the empty Ferrers diagram, denoted by $\varnothing$; the largest element is $\Gamma_{n}$ itself.

In what follows whenever we consider matrices with indices in $\mathbf{A}_{n}$, we shall assume that an arbitrary total order which refines $\subset$ has been chosen. Accordingly, an upper triangular matrix $M_{\alpha \beta}$ is a matrix such that $M_{\alpha \beta}=0$ whenever $\alpha \not \subset \beta$.

Finally, note that viewed as Ferrers diagrams, we have $\mathbf{A}_{n} \subset \mathbf{A}_{n+1}$. This embedding, in terms of sequences, sends $\alpha=\left(\alpha_{0}, \ldots, \alpha_{n-1}\right)$ to $\left(0, \alpha_{0}+1, \ldots, \alpha_{n-1}+1\right)$. One must be warned that not all quantities defined below satisfy a "stability" property with respect to this embedding i.e. some quantities depend explicitly on $n$ and not just on the underlying Ferrers diagrams. The quantities that are stable are the matrices $\mathbf{P}, \mathbf{C}, \mathcal{C}, \mathcal{I}$ to be defined below. The quantities that are not stable satisfy instead recurrence relations with respect to $n$, see Section 5.4.

\subsection{Schur functions}

To any Ferrers diagrams one can associate a Schur function. In the case of $\alpha \in \mathbf{A}_{n}$, and using an alphabet of $n$ letters, the Schur function can be defined in terms of the corresponding sequence of integers $\alpha=\left(\alpha_{i}\right)$ as

$$
s_{\alpha}(u)=\frac{\operatorname{det}\left(u_{i}^{\alpha_{j}}\right)_{0 \leqslant i, j \leqslant n-1}}{\Delta(u)}
$$

where $u:=\left(u_{0}, \ldots, u_{n-1}\right)$ and the denominator is simply the numerator with empty Ferrers diagram: $\Delta(u):=\prod_{0 \leqslant i<j \leqslant n-1}\left(u_{j}-u_{i}\right)$, that is the Vandermonde determinant. 


\subsection{The involution}

The Schur functions associated to $\alpha \in \mathbf{A}_{n}$ span a vector space of dimension the Catalan number $c_{n}=(2 n) ! / n ! /(n+1)$ !. It can be made into a commutative algebra $\Lambda_{n}$ by defining its structure constants to be the Littlewood-Richardson coefficients $\mathcal{C}_{\sigma \tau}^{\rho}$. In other words $\Lambda_{n}$ is a truncation of the algebra of symmetric functions in which one restricts oneself to diagrams inside $\Gamma_{n}=(n-1, n-2, \ldots, 1)$ : if $\Lambda_{\infty}$ denotes the algebra of symmetric functions, that is simply the algebra of all Schur functions with the ordinary function product, then $\Lambda_{n}$ is canonically identified with the quotient $\Lambda_{\infty}$ by the span of the $\sigma \not \subset \Gamma_{n}$, the latter being an ideal. In what follows we actually need the slightly larger space $\hat{\Lambda}_{\infty}$ of symmetric power series. $\Lambda_{n}$ is clearly also a quotient of $\hat{\Lambda}_{\infty}$.

We now introduce an involution $\imath$ on $\hat{\Lambda}_{\infty}$. It is defined by its action on elementary symmetric functions $e_{i}$ through their generating series $\prod_{i}\left(1+z u_{i}\right)=\sum_{i} e_{i} z^{i}$ : [14]

$$
\imath\left(\prod_{i}\left(1+z u_{i}\right)\right)=\prod_{i} \frac{1}{1-z \frac{u_{i}}{1+u_{i}}}
$$

where the $\left(u_{i}\right)$ are an arbitrary alphabet, and the equality should be understood order by order in $z$. By the morphism property this defines $\imath$ entirely on $\Lambda_{\infty}$ (we shall extend it below to $\left.\hat{\Lambda}_{\infty}\right)$.

Denote

$$
\tilde{s}_{\alpha}(u):=\imath\left(s_{\alpha}(u)\right)
$$

One can compute $\tilde{s}_{\alpha}(u)$ explicitly as follows. Note that by $(2.2), \imath$ is the composition of: (a) the change of variables $u \mapsto \frac{u}{1+u}$, and (b) the transposition of diagrams. ${ }^{1}$ Thus, if one defines the sequence $\left(\alpha_{i}^{\prime}\right)$ associated to the transpose diagram $\alpha^{\prime}$ as the ordered complement of the $\left\{2 n-1-\alpha_{i}\right\}$ inside $\{0,1, \ldots, 2 n-1\}$ (it can also be defined from the lengths of the columns $\tilde{\alpha}_{i}^{\prime}$ by $\left.\alpha_{i}^{\prime}=\tilde{\alpha}_{n-i}^{\prime}+i\right)$, then from (2.1),

$$
\begin{aligned}
\tilde{s}_{\alpha}(u) & =\frac{\operatorname{det}\left(\left(\frac{u_{i}}{1+u_{i}}\right)^{\alpha_{j}^{\prime}}\right)_{0 \leqslant i, j \leqslant n-1}}{\Delta\left(\frac{u}{1+u}\right)} \\
& =\prod_{i=0}^{n-1}\left(1+u_{i}\right)^{n-1} \frac{\operatorname{det}\left(\left(\frac{u_{i}}{1+u_{i}}\right)^{\alpha_{j}^{\prime}}\right)_{0 \leqslant i, j \leqslant n-1}}{\Delta(u)}
\end{aligned}
$$

This also leads to the following lemma:

Lemma 1. There is an expansion of the form

$$
\tilde{s}_{\alpha}(u)=s_{\alpha^{\prime}}(u)+\sum_{\beta \supsetneq \alpha^{\prime}} c_{\beta} s_{\beta}(u)
$$

where the $c_{\beta}$ are some coefficients.

\footnotetext{
${ }^{1}$ Equivalently, it is the composition of two commuting involutions: (a) $u \mapsto-\frac{u}{1+u}$ and (b) transposition of Ferrers diagrams composed with multiplication by $(-1)^{|\alpha|}$.
} 
Proof. Expand by multilinearity

$$
\frac{\operatorname{det}\left(\left(\frac{u_{i}}{1+u_{i}}\right)^{\alpha_{j}^{\prime}}\right)_{0 \leqslant i, j \leqslant n-1}}{\Delta(u)}=\sum_{k_{1}, \ldots, k_{n} \geqslant 0} c_{k_{1}, \ldots, k_{n}} \frac{\operatorname{det}\left(u_{i}^{\alpha_{j}^{\prime}+k_{j}}\right)_{0 \leqslant i, j \leqslant n-1}}{\Delta(u)}
$$

where the $c_{k_{1}, \ldots, k_{n}}$ are certain binomial coefficients, with $c_{0, \ldots, 0}=1$.

Note that the sequence $\left(\alpha_{i}^{\prime}+k_{i}\right)_{i=0, \ldots, n-1}$ is not necessarily increasing; however, if two terms are equal, then the determinant is zero, and if they are all distinct, then there exists a permutation $\mathcal{P}$ which reorders them; call $\beta$ the corresponding increasing sequence: $\beta_{\mathcal{P}(i)}:=\alpha_{i}^{\prime}+k_{i}$. In the latter case we have $\operatorname{det}\left(u_{i}^{\beta_{j}}\right) / \Delta(u)=(-1)^{|\mathcal{P}|} s_{\beta}(u)$.

Next we use the fact, which will be needed again in what follows, that if $\alpha_{i}^{\prime} \leqslant \beta_{\mathcal{P}(i)}$ for some $\mathcal{P}$ and all $i$, where $\alpha^{\prime}$ and $\beta$ are increasing sequences, then $\alpha_{i}^{\prime} \leqslant \beta_{i}$ for all $i$ (induction on the number of inversions $|\mathcal{P}|$, noting that if $i$ is such that $\mathcal{P}(i)>\mathcal{P}(i+1)$, then $\alpha_{i}^{\prime}<\alpha_{i+1}^{\prime} \leqslant \beta_{\mathcal{P}(i+1)}<\beta_{\mathcal{P}(i)}$, so that one can permute the images of $i$ and $i+1$, thus reducing $|\mathcal{P}|$ by one).

Combining the facts above leads to the expansion of the form of the lemma.

This lemma has two consequences. The first is that $\imath$ is well-defined on the whole of $\hat{\Lambda}_{\infty}$ (only finite sums occur for any coefficient of the image of any symmetric power series). It is then easy to check that $\imath$ is indeed an involution on $\hat{\Lambda}_{\infty}$.

The second is that this involution $\imath$ is compatible with the quotient to $\Lambda_{n}$ (keeping in mind that $\Gamma_{n}$ is invariant by transposition).

Finally, note that setting $z=1$ in (2.2) leads to

$$
\imath\left(\prod_{i}\left(1+u_{i}\right)\right)=\prod_{i}\left(1+u_{i}\right)
$$

Thus, $\prod_{i}\left(1+u_{i}\right)$, the sum of elementary symmetric functions, is left invariant by $\imath$.

\subsection{Change of basis}

The link patterns can be considered as forming the canonical basis of a vector space. It is however convenient to introduce another basis; it is naturally indexed by elements of $\mathbf{A}_{n}$ (increasing sequences) too, and is related to the canonical basis by a triangular change of basis (recall that we identify indices using the bijection of 2.1). If a vector has entries $\psi_{\pi}$ in the canonical basis and entries $\Psi_{\alpha}$ in this new basis (note the use of lowercase vs uppercase in order to distinguish these quantities), then

$$
\Psi_{\alpha}=\sum_{\pi \in \mathbf{A}_{n}} \psi_{\pi} \mathbf{P}^{\pi}{ }_{\alpha}
$$

Here $\mathbf{P}$ is the transpose of the usual matrix of change of basis. The reason for this transposition is that, to conform with the conventions of [22], our operators will act on the right. The matrix $\mathbf{P}$ is given explicitly in appendix B; we only need the fact that it is upper triangular, with ones on the diagonal. Its coefficients are closely connected with Kazhdan-Lusztig polynomials, see for example [21]. 

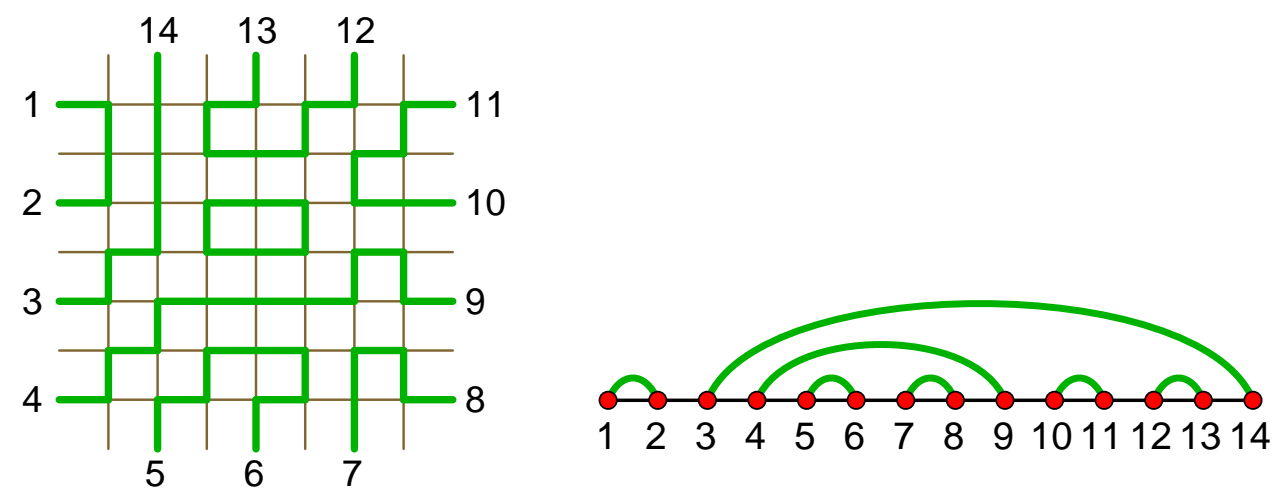

Figure 2: A FPL configuration and the associated link pattern.

\section{Statistical models of loops and Razumov-Stroganov conjecture}

\subsection{Counting of FPLs}

We introduce here the statistical lattice model called Fully Packed Loop (FPL) model. It is defined on a subset of the square lattice; in any given configuration of the model, edges of the lattice can have two states, empty or occupied, in such a way that each vertex has exactly two neighboring occupied edges (i.e. paths made of occupied edges visit every vertex of the lattice). We only consider the situation in which the Boltzmann weights are trivial, that is the pure enumeration problem.

Given a positive integer $n$, we are interested in FPL configurations inside an $n \times n$ grid with specific boundary conditions exemplified in Fig. 2: external edges are alternatingly occupied or empty. The justification for these boundary conditions comes from the connection to the six-vertex model (in which they correspond to the so-called Domain Wall Boundary Conditions), as well as to Alternating Sign Matrices, see [18].

Observe that there are two types of paths: the closed ones (loops) and the open ones, whose endpoints lie on the boundary. Ignoring the former, we see that to each FPL we can associate a link pattern that encodes the connectivity of its endpoints. Let us call $\psi_{\pi}$ the number of FPLs with connectivity $\pi$. Note that the endpoints must be labelled, which implies the choice of an origin; but it is in fact irrelevant due to Wieland's theorem [23], which states that $\psi_{\pi}=\psi_{\rho(\pi)}$ where $\rho(\pi)$ is the link pattern obtained from $\pi$ by cyclic rotation of the $2 n$ points.

In general, one does not know how to compute $\psi_{\pi}$. There has been however some progress [5, 11, 12, 4, 22], and we are particularly interested here in the formulae of $[4,22]$, which appear as a byproduct of proofs or attempted proofs of certain conjectures of [25]. Specifically, consider as in [22] FPL configurations in a triangle of the form of Fig. 3, with exactly $2 n$ vertical occupied external edges at the bottom, interlaced with $2 n-1$ empty edges. We further require that each of the $2 n-2$ external horizontal edges on the left (excluding the bottommost one) be connected to one of the $2 n-2$ external horizontal edges on the right. These configurations can be classified as follows: 


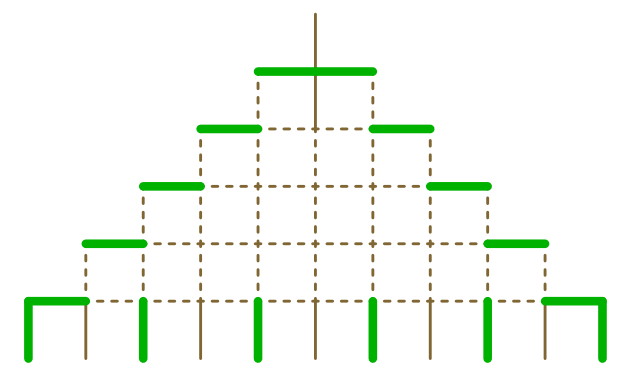

Figure 3: Boundary conditions for FPLs in a triangle.
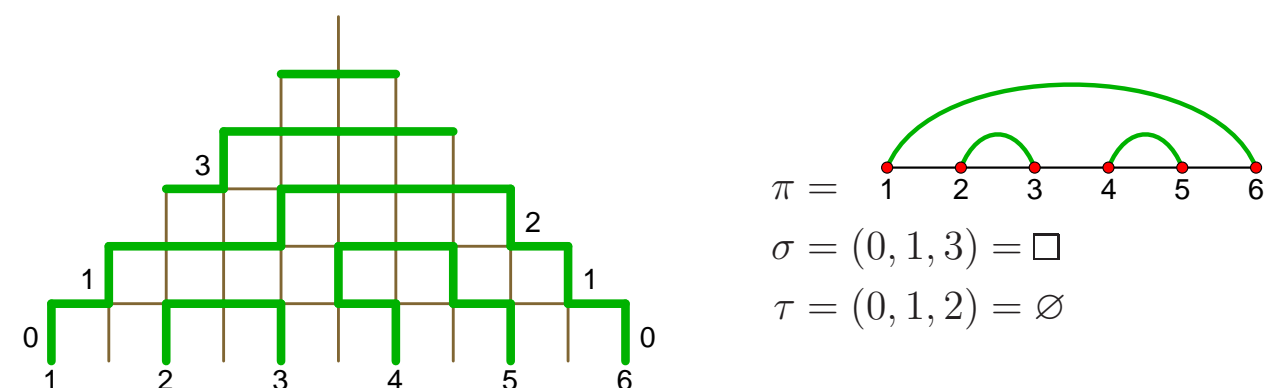

Figure 4: Example of parameterization of the boundary conditions of FPLs in a triangle.

the connectivity of the vertical external edges can be encoded into a link pattern $\pi$ of size $2 n$; furthermore, it is shown in [22] that if one considers the sequence of the $2 n$ vertical edges on either left or right boundaries read from bottom to top, then it forms a Dyck path with occupied=up and empty=down. Equivalently, in our language, the sequence of locations of occupied vertical edges on the left (resp. right) boundary, numbered from bottom (0) to top $(2 n-1)$, is a sequence in $\mathbf{A}_{n}$, which we denote by $\sigma$ (resp. $\tau$ ), see Fig. 4 for an example. Finally, define $a_{\sigma, \pi, \tau}$ to be the number of FPLs in a triangle with the boundary conditions defined above and given $\sigma, \pi, \tau$.

Then the following equality between the two enumeration problems holds:

$$
\psi_{\pi}=\sum_{\sigma, \tau \in \mathbf{A}_{n}} a_{\sigma, \pi, \tau} P_{\sigma^{\prime}}(-k) P_{\tau^{\prime}}(k-n+1)
$$

where $k$ is an integer to be discussed below, and $P_{\sigma}(x)$ is a polynomial of $x$ which, for positive integer $x$, coincides with the number of Semi-Standard Young Tableaux of shape $\sigma$; in fact,

$$
P_{\sigma}(x)= \begin{cases}s_{\sigma}(\underbrace{1, \ldots, 1}_{x}) & x \in \mathbb{Z}_{+} \\ s_{\sigma^{\prime}}(\underbrace{-1, \ldots,-1}_{-x}) & x \in \mathbb{Z}_{-}\end{cases}
$$

Explicitly, it is given by

$$
P_{\sigma}(x)=\prod_{i=0}^{n-1} \frac{(x-n+i+1) \cdots\left(x-n+\sigma_{i}\right)}{\sigma_{i} !} \prod_{0 \leqslant i<j \leqslant n-1}\left(\sigma_{j}-\sigma_{i}\right)
$$


Formula (3.1) can be deduced from Eq. (4) in [22]. ${ }^{2}$ In the derivation, the value of $k$ appears in relation to the geometry of FPLs, and the exact range of $k$ for which the formula is proved is not made clear. In the present work we only require that the formula be true for one value of $k$ - the explicit value being irrelevant since the result should be independent of $k$. See also Theorem 4.2 of [4] (which is the special case $k=0$ ).

\subsection{The Temperley-Lieb(1) loop model}

Another, a priori unrelated model is the following. Consider the semi-group generators $\mathbf{e}_{i}, 1 \leqslant i \leqslant 2 n$, acting on link patterns of size $2 n$ as follows: $\mathbf{e}_{i}$ turns a link pattern $\pi$ into the link pattern obtained from $\pi$ by pairing together (i) the points which are connected to $i$ and $i+1$ in $\pi$ and (ii) $i$ and $i+1$, all the other pairings remaining the same. For $i=2 n$ one assumes periodic boundary conditions i.e. $2 n+1 \equiv 1$. By linearity the $\mathbf{e}_{i}$ can be made into operators on the space of linear combinations of link patterns (thus forming a representation of the Temperley-Lieb(1) algebra, see [5] for more details) and one can then define the Hamiltonian:

$$
\mathbf{H}=\sum_{i=1}^{2 n} \mathbf{e}_{i}
$$

The $\mathbf{e}_{i}$ (resp. $\left.\mathbf{H}\right)$ possess a left eigenvector which is $(1, \ldots, 1)$ in the canonical basis, with eigenvalue 1 (resp. $2 n$ ); thus, $\mathbf{H}$ also possesses a (right) eigenvector with the same eigenvalue, denoted by $\psi^{\prime}$ :

$$
\mathbf{H} \psi^{\prime}=2 n \psi^{\prime}
$$

It is easy to check that $\mathbf{H}$ satisfies the hypotheses of the Perron-Frobenius theorem, so that $2 n$ is the (strictly) largest eigenvalue of $\mathbf{H}$, and $\psi^{\prime}$ is uniquely defined by (3.2) up to normalization. The latter, since $\mathbf{H}$ has integer entries, can always be chosen such that $\psi^{\prime}$ has positive coprime integer entries, denoted by $\psi_{\pi}^{\prime}$. An example is provided in appendix C.

In a series of papers $[7,8,10]$, it was shown how to generalize the Temperley-Lieb(1) loop model to an inhomogeneous model, then relate its Perron-Frobenius eigenvector to the quantum Knizhnik-Zamolodchikov equation, and finally write quasi-generating functions for entries of $\psi^{\prime}$. More precisely, the last step involves first performing the change of basis (2.5); then the new entries $\Psi_{\alpha}^{\prime}$ can be written

$$
\Psi_{\alpha}^{\prime}=\left.\Delta(u) \prod_{0 \leqslant i<j \leqslant n-1}\left(1+u_{j}+u_{i} u_{j}\right)\right|_{\prod_{i=0}^{n-1} u_{i}^{\alpha_{i}}}
$$

where $\mid . .$. means picking the coefficient of a monomial in a polynomial of the variables $u_{0}, \ldots, u_{n-1}$. (note that in [10] a slightly different notation $a_{i}=1+\alpha_{i-1}, 1 \leqslant i \leqslant n$ is used).

\footnotetext{
${ }^{2}$ Technically, it is obtained from Eq. (4) of [22] by setting $m=0$ in it, $m$ being the number of extra arches that surround all arches (see also Section 5.4 of the present work). The formula of Theorem 4.2 of [4] is only proved for $m$ sufficiently large, but a clever argument in Section 5 of [4] shows a property of polynomiality in $m$, which allows to continue it to $m=0$.
} 


\subsection{The Razumov-Stroganov conjecture}

Finally, to conclude this introductive section, we mention the following conjecture as inspiration for this work:

Conjecture. (Razumov, Stroganov [19]) For $\pi$ a link pattern of size $2 n$, let $\psi_{\pi}^{\prime}$ be as above the entry of the properly normalized Perron-Frobenius eigenvector of the Hamiltonian of the Temperley-Lieb(1) loop model, and $\psi_{\pi}$ be the number of FPLs with connectivity $\pi$; then

$$
\psi_{\pi}^{\prime}=\psi_{\pi}
$$

In a recent preprint [2], Cantini and Sportiello have proved this conjecture.

\section{The formula}

Let $n$ be a fixed positive integer, $\sigma, \tau$ be two Ferrers diagrams and $\alpha=\left(\alpha_{i}\right)$ be a sequence of integers in $\mathbf{A}_{n}$. We define $A_{\sigma, \alpha, \tau}$ to be the coefficient of a monomial in the expansion of a certain formal power series:

$$
A_{\sigma, \alpha, \tau}=\left.\tilde{s}_{\sigma}(u) s_{\tau}(u) \Delta(u) \prod_{i=0}^{n-1}\left(1+u_{i}\right)^{n-1} \prod_{0 \leqslant i<j \leqslant n-1}\left(1+u_{j}+u_{i} u_{j}\right)\right|_{\prod_{i=0}^{n-1} u_{i}^{\alpha_{i}}}
$$

Note the important fact that $\prod_{0 \leqslant i<j \leqslant n-1}\left(1+u_{j}+u_{i} u_{j}\right)$ is a nonsymmetric factor. If it were symmetric, we would simply be picking one term in the expansion of a certain symmetric function in terms of Schur functions, but it is not so.

One can rewrite (4.1) as a multiple contour integral in which the contours surround 0 clockwise (but not -1 ):

$$
A_{\sigma, \alpha, \tau}=\oint \prod_{i=0}^{n-1} \frac{d u_{i}}{2 \pi i u_{i}^{\alpha_{i}+1}} \tilde{s}_{\sigma}(u) s_{\tau}(u) \Delta(u) \prod_{i=0}^{n-1}\left(1+u_{i}\right)^{n-1} \prod_{0 \leqslant i<j \leqslant n-1}\left(1+u_{j}+u_{i} u_{j}\right)
$$

Using (2.1) and (2.3), one can also rewrite (4.1) more explicitly:

$$
A_{\sigma, \alpha, \tau}=\left.\frac{\operatorname{det}\left(u_{i}^{\tau_{j}}\right) \operatorname{det}\left(\left(\frac{u_{i}}{1+u_{i}}\right)^{\sigma_{j}^{\prime}}\right)}{\Delta(u)} \prod_{i=0}^{n-1}\left(1+u_{i}\right)^{2(n-1)} \prod_{0 \leqslant i<j \leqslant n-1}\left(1+u_{j}+u_{i} u_{j}\right)\right|_{\prod_{i=0}^{n-1} u_{i}^{\alpha_{i}}}
$$

where the $\tau_{j}$ and $\sigma_{j}^{\prime}$ are the increasing sequences associated to $\tau$ and $\sigma^{\prime}$.

In what follows we shall use the simplifying notation: let us write

$$
\langle F(u)\rangle_{\alpha}:=\left.F(u) \Delta(u) \prod_{0 \leqslant i<j \leqslant n-1}\left(1+u_{j}+u_{i} u_{j}\right)\right|_{\prod_{i=0}^{n-1} u_{i}^{\alpha_{i}}}
$$


for any symmetric function $F$. With this notation,

$$
A_{\sigma, \alpha, \tau}=\left\langle\tilde{s}_{\sigma}(u) s_{\tau}(u) \prod_{i=0}^{n-1}\left(1+u_{i}\right)^{n-1}\right\rangle_{\alpha}
$$

$A_{\sigma, \alpha, \tau}$ is of course an integer.

\subsection{Some properties}

An important fact is the following:

Lemma 2. If $|\sigma|+|\tau|>|\alpha|, A_{\sigma, \alpha, \tau}=0$. If $|\sigma|+|\tau|=|\alpha|, A_{\sigma, \alpha, \tau}=\mathcal{C}_{\sigma^{\prime}, \tau}^{\alpha}$.

Proof. By degree counting. According to Lemma 1, the lowest degree terms of (4.1) as a power series in the variables $u_{i}$ are $s_{\sigma^{\prime}}(u) s_{\tau}(u) \Delta(u)$. i.e. of degree $|\sigma|+|\tau|+n(n-1) / 2$. The first result follows from the fact that we pick out a term of degree $\sum_{i} \alpha_{i}=|\alpha|+n(n-1) / 2$. If $|\sigma|+|\tau|=|\alpha|$, one finds $A_{\sigma, \alpha, \tau}=s_{\sigma^{\prime}}(u) s_{\tau}(u) \Delta(u)_{\mid \prod_{i} u_{i}^{\alpha_{i}}}$. Expanding $s_{\sigma^{\prime}}(u) s_{\tau}(u) \Delta(u)=$ $\sum_{\rho} \mathcal{C}_{\sigma^{\prime}, \tau}^{\rho} s_{\rho}(u) \Delta(u)=\sum_{\rho} \mathcal{C}_{\sigma^{\prime}, \tau}^{\rho} \operatorname{det}\left(u_{i}^{\rho_{j}}\right)$, we conclude that $A_{\sigma, \alpha, \tau}=\mathcal{C}_{\sigma^{\prime}, \tau}^{\alpha}$.

Compare the first part of the lemma with Lemma 3.7 of [22]. By triangularity of the change of basis $\mathbf{P}$, the second part of the lemma also says that $a_{\sigma, \alpha, \tau}=\mathcal{C}_{\sigma^{\prime}, \tau}^{\alpha}$. This generalizes Lemma 3.6(b) of [22], that is Lemma 4.1(2) of [4].

Similarly, we have

Lemma 3. If $\tau \not \subset \alpha$ or $\sigma^{\prime} \not \subset \alpha, A_{\sigma, \alpha, \tau}=0$.

Proof. By symmetrizing the integrand of (4.2), one can write

$$
A_{\sigma, \alpha, \tau}=\oint \prod_{i=0}^{n-1} \frac{d u_{i}}{2 \pi i u_{i}} \tilde{s}_{\sigma}(u) s_{\tau}(u) \Delta(u) \prod_{i=0}^{n-1}\left(1+u_{i}\right)^{n-1} \mathrm{AS}\left[\frac{\prod_{0 \leqslant i<j \leqslant n-1}\left(1+u_{j}+u_{i} u_{j}\right)}{\prod_{i=0}^{n-1} u_{i}^{\alpha_{i}}}\right]_{\leqslant 0}
$$

where $\operatorname{AS}(f):=\frac{1}{n !} \sum_{\mathcal{P} \in \mathcal{S}_{n}}(-1)^{|\mathcal{P}|} f\left(u_{\mathcal{P}(1)}, \ldots, u_{\mathcal{P}(n)}\right)$, and $\leqslant 0$ means that one keeps only terms containing solely nonpositive powers of the $u_{i}$ since otherwise they do not contribute to the contour integral. Now expanding the product in brackets we notice that all monomials are of the form $\prod_{i} u_{i}^{-\beta_{i}}$ where $0 \leqslant \beta_{i} \leqslant \alpha_{i}$ for all $i$ :

$$
\mathrm{AS}\left[\frac{\prod_{0 \leqslant i<j \leqslant n-1}\left(1+u_{j}+u_{i} u_{j}\right)}{\prod_{i=0}^{n-1} u_{i}^{\alpha_{i}}}\right]_{\leqslant 0}=\sum_{0 \leqslant \beta_{i} \leqslant \alpha_{i}} c_{\beta} \operatorname{det}\left(u_{i, j}^{-\beta_{j}}\right)
$$

where the $c_{\beta}$ are some coefficients. Using the same reordering argument of the $\beta_{j}$ as in Lemma 1 (and absorbing the resulting sign in the $c_{\beta}$ ), we conclude that

$$
\begin{aligned}
A_{\sigma, \alpha, \tau} & =\sum_{\beta \subset \alpha} c_{\beta} \oint \prod_{i=0}^{n-1} \frac{d u_{i}}{2 \pi i u_{i}} \tilde{s}_{\sigma}(u) s_{\tau}(u) s_{\beta}\left(u^{-1}\right) \prod_{i=0}^{n-1}\left(1+u_{i}\right)^{n-1} \Delta(u) \Delta\left(u^{-1}\right) \\
& =\sum_{\beta \subset \alpha} c_{\beta}\left(\tilde{s}_{\sigma}(u) s_{\tau}(u) \prod_{i=0}^{n-1}\left(1+u_{i}\right)^{n-1} \mid s_{\beta}(u)\right)
\end{aligned}
$$


where by definition $\left(X(u) \mid s_{\beta}(u)\right)$ is the coefficient of $s_{\beta}(u)$ in the expansion of $X(u)$ as a sum of Schur functions. To perform this expansion, we use Lemma 1 and the well-known property of the multiplication of Schur functions that the resulting Ferrers diagrams must always contain the original ones, that is here $\sigma^{\prime}$ and $\tau$. We then find that non-zero terms are of the form $\sigma^{\prime}, \tau \subset \beta \subset \alpha$.

Compare with Lemma 4.1(1) of [4], also present as Lemma 3.6(a) in [22]. As a corollary, when $\sigma \notin \mathbf{A}_{n}$ or $\tau \notin \mathbf{A}_{n}, A_{\sigma, \alpha, \tau}=0$. From now on, we shall consider $A_{\sigma, \alpha, \tau}$ as a tensor where all three indices live in $\mathbf{A}_{n}$.

\subsection{Connection to FPLs}

The introduction of these quantities is motivated by

Conjecture 1. $\sum_{\alpha \in \mathbf{A}_{n}} A_{\sigma, \alpha, \tau}\left(\mathbf{P}^{-1}\right)_{\pi}^{\alpha}$ is equal to $a_{\sigma, \pi, \tau}$, the number of FPL configurations in a triangle with boundary conditions specified by $\sigma, \pi, \tau$ (cf. Section 3.1).

This conjecture has been checked numerically up to $n=5$, using the numerical data kindly provided by the author of [22].

\subsection{Summation}

We now show how summing the $A_{\sigma, \alpha, \tau}$ according to the prescription of [22] produces a formula which was proved in [10] in the context of the $q \mathrm{KZ}$ equation, that is on the other side of the Razumov-Stroganov conjecture.

Proposition 1. Conj. 1 implies the Razumov-Stroganov conjecture.

Proof. If Conj. 1 is true, then we can compute the number of FPL configurations with a given connectivity as follows. Fix an arbitrary integer $k$ and define

$$
\Psi_{\alpha}:=\sum_{\sigma, \tau \in \mathbf{A}_{n}} A_{\sigma, \alpha, \tau} P_{\sigma^{\prime}}(-k) P_{\tau^{\prime}}(k-n+1)
$$

(compare with (3.1)).

Inserting the formula (4.5) for $A_{\sigma, \alpha, \tau}$ yields

$$
\Psi_{\alpha}=\left\langle\prod_{i=0}^{n-1}\left(1+u_{i}\right)^{n-1} \sum_{\sigma \in \mathbf{A}_{n}} \tilde{s}_{\sigma}(u) P_{\sigma^{\prime}}(-k) \sum_{\tau \in \mathbf{A}_{n}} s_{\tau}(u) P_{\tau^{\prime}}(k-n+1)\right\rangle_{\alpha}
$$

The summations over $\sigma$ and $\tau$ can be easily performed (for example by use of the dual Cauchy identity or usual Cauchy identity depending on the sign of the arguments of $P$ ). We find $\sum_{\tau \in \mathbf{A}_{n}} s_{\tau}(u) P_{\tau^{\prime}}(k-n+1)=\prod_{i}\left(1+u_{i}\right)^{k-n+1}$. Similarly $\sum_{\sigma \in \mathbf{A}_{n}} \tilde{s}_{\sigma}(u) P_{\sigma^{\prime}}(-k)=$ $\imath\left(\prod_{i}\left(1+u_{i}\right)^{-k}\right)$ but by $(2.4)$ we can remove $\imath$ and the formula simplifies to

$$
\Psi_{\alpha}=\langle 1\rangle_{\alpha}=\left.\Delta(u) \prod_{0 \leqslant i<j \leqslant n-1}\left(1+u_{j}+u_{i} u_{j}\right)\right|_{\prod_{i=0}^{n-1} u_{i}^{\alpha_{i}}}
$$


which coincides with the expression (3.3) for $\Psi_{\alpha}^{\prime}$.

At this stage, to produce the number of FPL configurations with connectivity $\pi$, we should then apply the matrix $\mathbf{P}^{-1}$ to the entries $\Psi_{\alpha}$ (by combining the Conj. 1 with formula (3.1)). However this is not necessary, since showing $\Psi_{\alpha}^{\prime}=\Psi_{\alpha}$ is equivalent to $\psi_{\pi}^{\prime}=\psi_{\pi}$.

\subsection{Special cases}

The trivial case is when $\alpha=\varnothing$, that is the sequence $(0,1,2, \ldots, n-1)$. Since $|\alpha|=0$ we find $A_{\sigma, \varnothing, \tau}=\delta_{\sigma, \varnothing} \delta_{\tau, \varnothing}$ and $\Psi_{\varnothing}=1$.

Another special case is when $\alpha=\Gamma_{n}$, the largest element of $\mathbf{A}_{n}$, that is the sequence $(0,2, \ldots, 2 n-2)$. In this case we can use the result first conjectured in [10] and proved in $[24,13]$ :

$$
\begin{aligned}
{\left[\prod_{0 \leqslant i \leqslant j \leqslant n-1}\left(1-u_{i} u_{j}\right) \operatorname{AS}\left(\prod_{i=0}^{n-1} u_{i}^{-2 i} \prod_{0 \leqslant i<j \leqslant n-1}\left(1+u_{i} u_{j}+\boldsymbol{\tau} u_{j}\right)\right)\right]_{\leqslant 0} } \\
=\operatorname{AS}\left(\prod_{i=0}^{n-1}\left(u_{i}^{-1}\left(\boldsymbol{\tau}+u_{i}^{-1}\right)\right)^{i}\right) \\
=\prod_{0 \leqslant i<j \leqslant n-1}\left(u_{j}^{-1}-u_{i}^{-1}\right)\left(\boldsymbol{\tau}+u_{i}^{-1}+u_{j}^{-1}\right)
\end{aligned}
$$

Here $\boldsymbol{\tau}=1$. The left hand side is exactly what appears in Eq. (4.6) for $\alpha=\Gamma_{n}$, the truncation to nonpositive monomials being possible since other terms do not contribute to the constant term. Replacing with the right hand sides gives us two expressions:

$$
\begin{aligned}
A_{\sigma, \Gamma_{n}, \tau}= & \left.\tilde{s}_{\sigma}(u) s_{\tau}(u) \Delta(u) \prod_{0 \leqslant i \leqslant j \leqslant n-1}\left(1-u_{i} u_{j}\right)^{-1} \prod_{i=0}^{n-1} u_{i}^{-2 i}\left(1+u_{i}\right)^{n+i-1}\right|_{C T} \\
= & \tilde{s}_{\sigma}(u) s_{\tau}(u) \Delta(u) \Delta\left(u^{-1}\right) \\
& \left.\prod_{0 \leqslant i \leqslant j \leqslant n-1}\left(1-u_{i} u_{j}\right)^{-1} \prod_{0 \leqslant i<j \leqslant n-1}\left(1+u_{i}^{-1}+u_{j}^{-1}\right) \prod_{i=0}^{n-1}\left(1+u_{i}\right)^{n-1}\right|_{C T}
\end{aligned}
$$

(where $C T$ means extracting the constant term). Equivalently, we have

$$
A_{\sigma, \Gamma_{n}, \tau}=\left(\tilde{s}_{\sigma}(u) s_{\tau}(u) \prod_{i=0}^{n-1}\left(1+u_{i}\right)^{n-1} \prod_{0 \leqslant i \leqslant j \leqslant n-1}\left(1-u_{i} u_{j}\right)^{-1} \mid \prod_{0 \leqslant i<j \leqslant n-1}\left(1+u_{i}+u_{j}\right)\right)
$$

where we have used as before the usual scalar product $(\cdot \mid \cdot)$ for which Schur functions are orthonormal. Note that both bra and ket depend explicitly on $n$.

It is shown in [10] by direct calculation that $\mathbf{P}_{\Gamma_{n}}^{\pi}=\delta_{\Gamma_{n}}^{\pi}$. Therefore, $a_{\sigma, \Gamma_{n}, \tau}=A_{\sigma, \Gamma_{n}, \tau}$ and the formula above gives a closed expression for it. 


\section{Analogues of Thapper's conjectures}

In this section we prove Thapper's conjectures assuming Conj. 1, that is we prove some

properties of the $A_{\sigma, \alpha, \tau}$ that are analogous to those found by Thapper in the context of FPL enumeration.

\subsection{The action of $\Lambda_{n}$}

Consider the operator that acts by inserting a factor $s_{\lambda}(u)$ in the generating series. It is convenient to introduce another bracket notation $\langle\cdot\rangle_{\sigma, \alpha, \tau}$ which, compared to $\langle\cdot\rangle_{\alpha}$, incorporates the factors $\tilde{s}_{\sigma}(u) s_{\tau}(u) \prod_{i=0}^{n-1}\left(1+u_{i}\right)^{n-1}$. In other words let us compute

$$
\left\langle s_{\lambda}(u)\right\rangle_{\sigma, \alpha, \tau}:=\left.s_{\lambda}(u) \tilde{s}_{\sigma}(u) s_{\tau}(u) \Delta(u) \prod_{i=0}^{n-1}\left(1+u_{i}\right)^{n-1} \prod_{0 \leqslant i<j \leqslant n-1}\left(1+u_{j}+u_{i} u_{j}\right)\right|_{\prod_{i=0}^{n-1} u_{i}^{\alpha_{i}}}
$$

Similarly consider the insertion of $\tilde{s}_{\lambda}(u)$ :

$$
\left\langle\tilde{s}_{\lambda}(u)\right\rangle_{\sigma, \alpha, \tau}:=\left.\tilde{s}_{\lambda}(u) \tilde{s}_{\sigma}(u) s_{\tau}(u) \Delta(u) \prod_{i=0}^{n-1}\left(1+u_{i}\right)^{n-1} \prod_{0 \leqslant i<j \leqslant n-1}\left(1+u_{j}+u_{i} u_{j}\right)\right|_{\prod_{i=0}^{n-1} u_{i}^{\alpha_{i}}}
$$

Note that one can expand $s_{\lambda}(u) s_{\tau}(u)$ in terms of Schur functions, resulting in

$$
\left\langle s_{\lambda}(u)\right\rangle_{\sigma, \alpha, \tau}=\sum_{\mu \in \mathbf{A}_{n}} \mathcal{C}_{\lambda, \tau}^{\mu} A_{\sigma, \alpha, \mu}
$$

where the summation can be restricted to $\mathbf{A}_{n}$ because all other terms vanish. Of course the matrices $\mathcal{C}(\lambda)_{\tau}^{\mu}:=\mathcal{C}_{\lambda, \tau}^{\mu}$ form a representation $\lambda \mapsto \mathcal{C}(\lambda)$ of $\Lambda_{n}$ (regular representation).

One can also expand $\tilde{s}_{\lambda}(u) s_{\tau}(u)$ in terms of Schur functions $s_{\mu}(u)$, with some a priori unknown coefficients $\tilde{\mathcal{C}}_{\lambda, \tau}^{\mu}$ :

$$
\left\langle\tilde{s}_{\lambda}(u)\right\rangle_{\sigma, \alpha, \tau}=\sum_{\mu \in \mathbf{A}_{n}} \tilde{\mathcal{C}}_{\lambda, \tau}^{\mu} A_{\sigma, \alpha, \mu}
$$

One must be careful that $\tilde{\mathcal{C}}_{\lambda, \tau}^{\mu}$ is not symmetric in the exchange of $\lambda$ and $\tau$. If we call $\tilde{\mathcal{C}}(\lambda)_{\tau}^{\mu}:=\tilde{\mathcal{C}}_{\lambda, \tau}^{\mu}$, this gives another distinct action of $\Lambda_{n}$. It commutes with the previous one.

In fact in what follows it will be more natural to consider the transpose matrices $\mathcal{C}(\lambda)^{T}$ and $\tilde{\mathcal{C}}(\lambda)^{T}$, which also form representations of $\Lambda_{n}$ (since it is commutative).

Of course we have dual statements by expanding this time the expression in brackets multiplied by $\tilde{s}_{\sigma}(u)$ in terms of $\tilde{s}_{\mu}(u)$ and using the fact that $\imath$ is an involution:

$$
\left\langle s_{\lambda}(u)\right\rangle_{\sigma, \alpha, \tau}=\sum_{\mu \in \mathbf{A}_{n}} \tilde{\mathcal{C}}_{\lambda, \sigma}^{\mu} A_{\mu, \alpha, \tau}
$$


and

$$
\left\langle\tilde{s}_{\lambda}(u)\right\rangle_{\sigma, \alpha, \tau}=\sum_{\mu \in \mathbf{A}_{n}} \mathcal{C}_{\lambda, \sigma}^{\mu} A_{\mu, \alpha, \tau}
$$

Finally we can also expand $s_{\lambda}(u) \prod u_{i}^{-\alpha_{i}}$ in monomials. This is actually more subtle than it seems because it results in monomials for which the sequence of inverse powers is not increasing. It is shown in appendix A (Lemma 4) that one can always reexpress the coefficient of any monomial as a linear combination of the coefficients of the monomials with increasing inverse powers. Note that we can discard any positive powers because they do not contribute.

That is, there exist coefficients $\mathbf{C}_{\lambda, \alpha}^{\beta}$ such that

$$
\left\langle s_{\lambda}(u) F(u)\right\rangle_{\alpha}=\sum_{\beta \in \mathbf{A}_{n}} \mathbf{C}_{\lambda, \alpha}^{\beta}\langle F(u)\rangle_{\beta}
$$

for any symmetric function $F$, and similarly for $\tilde{\mathbf{C}}_{\lambda, \alpha}^{\beta}$.

In the present case, we find

$$
\left\langle s_{\lambda}(u)\right\rangle_{\sigma, \alpha, \tau}=\sum_{\beta \in \mathbf{A}_{n}} \mathbf{C}_{\lambda, \alpha}^{\beta} A_{\sigma, \beta, \tau}
$$

and

$$
\left\langle\tilde{s}_{\lambda}(u)\right\rangle_{\sigma, \alpha, \tau}=\sum_{\beta \in \mathbf{A}_{n}} \tilde{\mathbf{C}}_{\lambda, \alpha}^{\beta} A_{\sigma, \beta, \tau}
$$

which can be made into matrices $\mathbf{C}(\lambda)^{\beta}{ }_{\alpha}:=\mathbf{C}_{\lambda, \alpha}^{\beta}$ and $\tilde{\mathbf{C}}(\lambda)^{\beta}{ }_{\alpha}:=\tilde{\mathbf{C}}_{\lambda, \alpha}^{\beta}$.

Combining these relations (5.3-5.9) results in various identities.

A special case occurs when one considers the multiplication by $\prod_{i}\left(1+u_{i}\right)$, because of the fact that it is invariant by the involution. We have:

$$
\left\langle\prod_{i}\left(1+u_{i}\right)\right\rangle_{\sigma, \alpha, \tau}=\sum_{\mu \in \mathbf{A}_{n}} \mathcal{C}_{\tau}^{\mu} A_{\sigma, \alpha, \mu}=\sum_{\mu \in \mathbf{A}_{n}} \mathcal{C}_{\sigma}^{\mu} A_{\mu, \alpha, \tau}=\sum_{\beta \in \mathbf{A}_{n}} \mathbf{C}_{\alpha}^{\beta} A_{\sigma, \beta, \tau}
$$

with the obvious notations $\mathcal{C}_{\tau}^{\mu}=\sum_{i=0}^{n-1} \mathcal{C}_{e_{i}, \tau}^{\mu}$ and $\mathbf{C}_{\alpha}^{\beta}=\sum_{i=0}^{n-1} \mathbf{C}_{e_{i}, \alpha}^{\beta}$ ( $e_{i}$ is the one-column diagram with $i$ boxes).

Observe also that taking $\sigma=\varnothing$ in (5.6) and combining with (5.4) results in

$$
A_{\lambda, \alpha, \tau}=\sum_{\mu \in \mathbf{A}_{n}} \tilde{\mathcal{C}}_{\lambda, \tau}^{\mu} A_{\varnothing, \alpha, \mu}
$$

\subsection{Matrix identities}

Introduce, following [22], the matrices $A(\lambda)_{\tau \alpha}:=A_{\lambda, \alpha, \tau}$ and $\bar{A}(\alpha)_{\sigma \tau}:=A_{\sigma, \alpha, \tau}$. Note that Lemma 3 says that the $A(\sigma)$ are upper triangular. We now rewrite the various identities of the previous section in these matrix notations. 
Relations (5.3-5.9) become

$$
\begin{aligned}
& \tilde{\mathcal{C}}(\lambda)^{T} \bar{A}(\alpha)=\bar{A}(\alpha) \mathcal{C}(\lambda) \\
& \mathcal{C}(\lambda)^{T} A(\sigma)=A(\sigma) \mathbf{C}(\lambda) \\
& \mathcal{C}(\lambda)^{T} \bar{A}(\alpha)=\bar{A}(\alpha) \tilde{\mathcal{C}}(\lambda) \\
& \tilde{\mathcal{C}}(\lambda)^{T} A(\sigma)=A(\sigma) \tilde{\mathbf{C}}(\lambda)
\end{aligned}
$$

and (5.10) becomes

$$
\begin{aligned}
\mathcal{C}^{T} \bar{A}(\alpha) & =\bar{A}(\alpha) \mathcal{C} \\
\mathcal{C}^{T} A(\alpha) & =A(\alpha) \mathbf{C}
\end{aligned}
$$

Compare with Conj. 3.4 and 3.5(a) of [22] (with $B=\mathcal{C}^{T}: B$ removes columns of boxes to Ferrers diagrams whereas $\mathcal{C}$ adds them - so that $B$ is upper triangular whereas $\mathcal{C}$ is lower triangular).

As to (5.11), it becomes

$$
A(\lambda)=\tilde{\mathcal{C}}(\lambda)^{T} A(\varnothing)
$$

According to Lemmas 3 and $2, A(\varnothing)$ is upper triangular with ones on the diagonal, and therefore invertible. The matrices $A(\lambda) A(\varnothing)^{-1}$ are equal to $\tilde{\mathcal{C}}(\lambda)^{T}$ and thus satisfy the relations of the $\Lambda_{n}$ algebra, cf. Prop. 3.9 of [22]. In particular they commute, cf. Prop. 3.10 of [22].

One can similarly combine (5.6) and (5.9) to obtain

$$
A(\lambda)=A(\varnothing) \tilde{\mathbf{C}}(\lambda)
$$

i.e. $\tilde{\mathbf{C}}(\lambda)=A(\varnothing)^{-1} A(\lambda)$.

More directly, we conclude from $(5.13,5.15)$ that the matrices $\mathbf{C}(\lambda)$ and $\tilde{\mathbf{C}}(\lambda)$ provide us with another pair of commuting representations of $\Lambda_{n}$; and that $A(\varnothing)$ intertwines the representations $\mathbf{C}(\lambda)$ and $\mathcal{C}(\lambda)^{T}$, as well as $\tilde{\mathbf{C}}(\lambda)$ and $\tilde{\mathcal{C}}(\lambda)^{T}$. In fact, any linear combination of the $A(\sigma)$ is an intertwiner, but for it to be invertible the coefficient of $A(\varnothing)$ must be non-zero.

\subsection{The involution}

Consider the matrix $\mathcal{I}$ of the involution $\imath$ acting on $\Lambda_{n}$ :

$$
\tilde{s}_{\lambda}=\sum_{\mu \in \mathbf{A}_{n}} \mathcal{I}_{\lambda}^{\mu} s_{\mu}+\text { terms not inside } \Gamma_{n}
$$

(equivalently $\mathcal{I}_{\lambda}^{\mu}:=\tilde{\mathcal{C}}_{\lambda, \varnothing}^{\mu}$ ). Then by definition we have

$$
\tilde{\mathcal{C}}(\lambda)=\mathcal{I C}(\lambda) \mathcal{I}
$$

That is, $\mathcal{I}$ intertwines the representations $\mathcal{C}(\lambda)$ and $\tilde{\mathcal{C}}(\lambda)$ of $\Lambda_{n}$. 
We now have a chain of (invertible) intertwiners between the four representations $\mathbf{C}(\lambda), \mathcal{C}(\lambda)^{T}, \tilde{\mathcal{C}}(\lambda)^{T}, \tilde{\mathbf{C}}(\lambda)$. Composing them, we find that

$$
\tilde{\mathbf{C}}(\lambda)=\mathbf{R C}(\lambda) \mathbf{R}
$$

with $\mathbf{R}:=A(\varnothing)^{-1} \mathcal{I}^{T} A(\varnothing), \mathbf{R}^{2}=1$.

What is the meaning of $\mathbf{R}$ ? It is a simple exercise to check, combining the various relations found so far, that

$$
\sum_{\beta \in \mathbf{A}_{n}} A_{\sigma, \beta, \tau} \mathbf{R}_{\alpha}^{\beta}=A_{\tau, \alpha, \sigma}
$$

That is, $\mathbf{R}$ corresponds to the operation of mirror image in the space of link patterns. In the canonical link pattern basis $\left(\mathbf{r}:=\mathbf{P R} \mathbf{P}^{-1}\right)$, we simply have $\mathbf{r}_{\pi^{\prime}}=1$ if $\pi$ and $\pi^{\prime}$ are mirror images of each other, and 0 otherwise.

\subsection{Recurrence}

Let $m$ be a non-negative integer. By definition, let $(\alpha)_{m}$ be the sequence of increasing integers in $\mathbf{A}_{n+m}$ associated to a sequence $\alpha \in \mathbf{A}_{n}$ by the embedding of $\mathbf{A}_{n}$ into $\mathbf{A}_{n+m}$ described in Section 2.2. Explicitly, $(\alpha)_{m}=\left(0,1, \ldots, m-1, m+\alpha_{0}, \ldots, m+\alpha_{n-1}\right)$. In other words, viewed as a Dyck path, $(\alpha)_{m}$ consists of $m$ up steps, then the Dyck path $\alpha$ of length $2 n$, then $m$ down steps. We also write $(\alpha)_{1}=(\alpha)$. Let us derive a recurrence relation for $\Psi_{(\alpha)_{m}}$ using the formalism of the previous sections.

Start with the formula (4.9) for $\Psi_{(\alpha)_{m}}$ and note that we are simply looking for the constant term as a function of $u_{0}$. So we can set $u_{0}=0$; as a result, the Vandermonde determinant shifts all powers of $u_{i}$ by 1 , so that one can now apply the same argument to $u_{1}$, etc. After $m$ steps we find the following formula:

$$
\Psi_{(\alpha)_{m}}=\left.\Delta(u) \prod_{i=0}^{n-1}\left(1+u_{i}\right)^{m} \prod_{0 \leqslant i<j \leqslant n-1}\left(1+u_{j}+u_{i} u_{j}\right)\right|_{\prod_{i=0}^{n-1} u_{i}^{\alpha_{i}}}
$$

Using bracket notations, this is nothing but

$$
\Psi_{(\alpha)_{m}}=\left\langle\prod_{i=0}^{n-1}\left(1+u_{i}\right)^{m}\right\rangle_{\alpha}
$$

Note that this means that $\Psi$ does not satisfy a stability property with respect to the embedding of $\mathbf{A}_{n}$ into $\mathbf{A}_{n+m}$.

The insertion of such products has already been analyzed in Section 5.1. Applying (5.7), we find a recurrence relation for $\Psi_{\alpha}$ :

$$
\Psi_{(\alpha)_{m}}=\sum_{\beta \in \mathbf{A}_{n}} \Psi_{\beta}\left(\mathbf{C}^{m}\right)_{\alpha}^{\beta}
$$

It formulates a component of size $m+n$ in terms of components of size $n$. 
Let us now discuss briefly the change of basis to the link pattern basis. It turns out to be "compatible" with the operation $(\cdot)_{m}$ in the following sense: for all $\rho \in \mathbf{A}_{n+m}, \alpha \in \mathbf{A}_{n}$,

$$
\mathbf{P}^{\rho}{ }_{(\alpha)_{m}}= \begin{cases}\mathbf{P}^{\pi}{ }_{\alpha} & \text { if } \rho=(\pi)_{m} \text { for some } \pi \in \mathbf{A}_{n} \\ 0 & \text { otherwise }\end{cases}
$$

(this property is a combination of the upper triangularity and stability with respect to the embedding of $\mathbf{P}$ ). Thus, writing

$$
\Psi_{(\alpha)_{m}}=\sum_{\pi \in \mathbf{A}_{n}} \Psi_{(\pi)_{m}} \mathbf{P}_{\alpha}^{\pi}
$$

and inverting $\mathbf{P}$, we can rewrite (5.26)

$$
\psi_{(\pi)_{m}}=\sum_{\rho \in \mathbf{A}_{n}} \psi_{\rho}\left(\mathbf{c}^{m}\right)^{\rho}{ }_{\pi}
$$

with $\mathbf{c}:=\mathbf{P C P}^{-1}$.

In particular, for $m=1$, since any link pattern contains a pairing of neighbors, which after appropriate rotation can be mapped to $(0,2 n-1),(5.29)$ provides a closed recurrence relation for the $\psi_{\pi}$.

NB: one can also write recurrence relations for the $A_{\sigma, \alpha, \tau}$. Following the same reasoning as for $\Psi$, but paying attention to the extra factors occurring because of the explicit dependence of $A_{\sigma,(\alpha)_{m}, \tau}$ on the size $m+n$, we find that $A_{\sigma,(\alpha)_{m}, \tau}=\left\langle\prod_{i=0}^{n-1}\left(1+u_{i}\right)^{2 m}\right\rangle_{\sigma, \alpha, \tau}$. Just like $\Psi, A_{\sigma, \alpha, \tau}$ does not satisfy a stability property with respect to the embedding of $\mathbf{A}_{n}$ into $\mathbf{A}_{n+m}$. Use of relations (5.10) give various identities, including recurrences, for the $A_{\sigma, \alpha, \tau}$. Also, if one uses one of the first two equalities of (5.10) and apply it to the relation (4.7), one finds

$$
\Psi_{(\alpha)_{m}}=\sum_{\sigma, \tau \in \mathbf{A}_{n}} A_{\sigma, \alpha, \tau} P_{\sigma^{\prime}}(2 m-k) P_{\tau^{\prime}}(k-n-m+1)
$$

or the same expression with instead $P_{\sigma^{\prime}}(-k) P_{\tau^{\prime}}(k-n+m+1)$, which is of course the same up to a change of the arbitrary integer $k$. This expression is Eq. (4) (with arbitrary $m$ ) of [22] (see also Theorem 4.2 of [4]).

\subsection{Largest component}

As has already been mentioned in Section 4.4, the change of basis is trivial for the largest component, so that the component $\psi_{\Gamma_{n}}$ (i.e. with $\Gamma_{n}$ viewed as a link pattern) is the same as the component $\Psi_{\Gamma_{n}}$ in our basis (i.e. the component with sequence $\alpha_{i}=2 i$ ).

Furthermore, it is also known [10] how to obtain the sum of components; one has

$$
\sum_{\epsilon_{1}, \ldots, \epsilon_{n-1} \in\{0,1\}} \mathbf{P}^{\pi}{ }_{\left(0,2-\epsilon_{1}, \ldots, 2(n-1)-\epsilon_{n-1}\right)}=1 \quad \forall \pi \in \mathbf{A}_{n}
$$


Note that this is exactly the image of $\Gamma_{n}$ by the action of $\mathbf{C}$, so that $(\mathbf{P C})^{\pi} \Gamma_{n}=1$ for all $\pi$. Multiplying on the right by $\mathbf{P}^{-1}$ and using again $\mathbf{P}_{\Gamma_{n}}^{\pi}=\delta_{\Gamma_{n}}^{\pi}$ we find $\mathbf{c}_{\Gamma_{n}}^{\pi}=1$, which is exactly the content of Conj. 3.5(b) of [22].

Thus, as explained in [22], the recurrence produces in this case the identity

$$
\psi_{\Gamma_{n+1}}=\sum_{\pi \in \mathbf{A}_{n}} \psi_{\pi}
$$

as well as the more general one

$$
\psi_{\left(\Gamma_{n}\right)_{m+1}}=\sum_{\pi \in \mathbf{A}_{n}} \psi_{(\pi)_{m}}
$$

to compare with the conjectures of [25].

\subsection{The A's from the $\Psi$ 's}

It it perhaps suggestive that using the various matrices defined above, one can in fact compute $A_{\sigma, \alpha, \tau}$ from the data of the $\Psi_{\alpha}$ alone. Indeed, introducing yet another notation $A(\sigma, \tau)$ for the row vector with entries $A_{\sigma, \alpha, \tau}$, and similarly for $\Psi$, we have

$$
A(\sigma, \tau)=\Psi \tilde{\mathbf{C}}(\sigma) \mathbf{C}(\tau) \mathbf{C}^{n-1}
$$

\section{The $\tau$-generalization}

In $[17,8]$, it was suggested how to generalize the ground state eigenvector $\psi^{\prime}$ of the Temperley-Lieb(1) model into a vector depending on an extra parameter $\boldsymbol{\tau}$ (often written as $\boldsymbol{\tau}=-q-q^{-1}$ ) and which is obtained by specializing a certain polynomial solution of the quantum Knizhnik-Zamolodchikov ( $q \mathrm{KZ}$ ) equation. The original vector is recovered by taking $\boldsymbol{\tau}=1$. The parameter $\boldsymbol{\tau}$ does not seem to have any obvious meaning in terms of FPLs, though a connection to Totally Symmetric Self-Complementary Plane Partitions was found in [6]. Many formulae generalize to $\boldsymbol{\tau}$ away from unity [10]. It is natural to wonder if the formulae of the present work fall in this category. We briefly present here this generalization.

Introduce the $\boldsymbol{\tau}$-dependent bracket

$$
\langle F(u)\rangle_{\alpha}:=\left.F(u) \Delta(u) \prod_{0 \leqslant i<j \leqslant n-1}\left(1+\boldsymbol{\tau} u_{j}+u_{i} u_{j}\right)\right|_{\prod_{i=0}^{n-1} u_{i}^{\alpha_{i}}}
$$

This produces a natural generalization of the $\Psi_{a}$ :

$$
\Psi_{a}=\langle 1\rangle_{\alpha}
$$

which corresponds to the homogeneous limit of a solution of the $q \mathrm{KZ}$ system of difference equations. The change of basis to the link pattern basis can be found for all $\boldsymbol{\tau}$ in [10] (or in (B.1) where the $U_{i}$ are Chebyshev polynomials of $\boldsymbol{\tau}$ ). 
Since the interpretation in terms of FPLs in a triangle is lacking, there seems little point in introducing the $\boldsymbol{\tau}$-generalization of $A_{\sigma, \alpha, \tau}$. However, one can still define the various matrices of multiplication by a Schur function $s_{\lambda}$. The matrices $\mathcal{C}(\lambda)$ are of course unchanged; however the matrices $\mathbf{C}(\lambda)$ which act on increasing sequences by

$$
\left\langle s_{\lambda}(u) F(u)\right\rangle_{\alpha}=\sum_{\beta \in \mathbf{A}_{n}} \mathbf{C}_{\lambda, \alpha}^{\beta}\langle F(u)\rangle_{\beta}
$$

now have entries which are polynomials in $\tau$, and can be computed using the methods of appendices A and B. They still form a reprentation of $\Lambda_{n}$. The dual versions can be defined via the involution

$$
\imath\left(\prod_{i}\left(1+z u_{i}\right)\right)=\prod_{i} \frac{1}{1-z \frac{u_{i}}{1+\tau u_{i}}}
$$

Of particular interest is the (self-dual) operator $\mathbf{C}$ of multiplication by $\prod_{i=0}^{n-1}\left(1+\boldsymbol{\tau} u_{i}\right)$, that is

$$
\mathbf{C}=\sum_{i=0}^{n-1} \mathbf{C}\left(e_{i}\right) \boldsymbol{\tau}^{i}
$$

It corresponds to setting $z=\boldsymbol{\tau}$ in the notations of appendix B.

Then all the recursion relations conjectured in [22] are satisfied by $\Psi$ for all $\boldsymbol{\tau}$, with the use of this modified matrix C. Namely, equations (5.26) and (5.29) hold without any change. However, naively, they do not form a closed set of recursion relations. The reason is that for $\boldsymbol{\tau} \neq 1$, Wieland's rotational invariance theorem does not hold any more: in general $\psi_{\pi} \neq \psi_{\rho(\pi)}$, so one cannot assume that there exists a pairing $(0,2 n-1)$.

However, a remarkable phenomenon occurs: in the link pattern basis, one has

$$
\psi_{\pi}=\sum_{\rho \in \mathbf{A}_{n}} \psi_{\rho} \mathbf{c}_{\pi}^{(\rho)}
$$

where now $\pi$ is an arbitrary link pattern of size $n+1$, and $\mathbf{c}$ is the matrix of size $c_{n+1}$. This is a non-trivial generalization of Eq. (5.29) at $m=1$, in the sense that in the special case where $\pi$ has a pairing $(0,2 n-1)$, i.e. $\pi=\left(\pi^{\prime}\right)$, we recover $(5.29)$.

Thus, we have a closed recursion again, and the vectors $\psi$ for successive values of $n$ can be obtained by simply iterating the matrices $\mathbf{c}$ in size $1, \ldots, n$. This observation will be the subject of future work.

Note added. After this work was completed, Nadeau announced [15] a direct proof of Conj. 3.4 of [22] i.e. the analogue of (5.17). He also announced [16] a bijection between Knutson-Tao puzzles and FPLs in a triangle with $|\alpha|=|\sigma|+|\tau|$, which would provide a bijective proof of the second part of Lemma 2. 


\section{A Existence of the matrix $\mathrm{K}$}

In this section we no longer assume that the sequences $\left(\alpha_{i}\right)$ are increasing.

Lemma 4. Let $\alpha=\left(\alpha_{0}, \ldots, \alpha_{n-1}\right)$ be an arbitrary sequence of integers such that $\alpha_{i} \leqslant 2 i$. There exist coefficients $\mathbf{K}_{\alpha}^{\beta}$ such that for any symmetric function $F(u)$,

$$
\langle F(u)\rangle_{\alpha}=\sum_{\beta \in \mathbf{A}_{n}}\langle F(u)\rangle_{\beta} \mathbf{K}_{\alpha}^{\beta}
$$

Proof. The relation can be checked on Schur functions $s_{\tau}(u)$ only. Furthermore, if $\tau \notin \mathbf{A}_{n}$, because $\alpha_{i} \leqslant 2 i$, both sides of the equality are zero. So one can think of $\langle\cdot\rangle_{\alpha}$ as a linear form on $\Lambda_{n}$. It it actually convenient at this stage to switch to a different basis of $\Lambda_{n}$, namely $s_{\tau}(u) \prod_{i=0}^{n-1}\left(1+u_{i}\right)^{n-1}$ (in order to avoid having to introduce yet another new notation). Plugging this into (A.1), we note that the right hand side is simply $(A(\varnothing) \mathbf{K})_{\tau, \alpha}$. Let us thus define

$$
A^{e x t}(\varnothing)_{\tau, \alpha}=\left\langle s_{\tau}(u) \prod_{i=0}^{n-1}\left(1+u_{i}\right)^{n-1}\right\rangle_{\alpha}
$$

that is the same definition as for $A$, but in which we relax the constraint that $\left(\alpha_{i}\right)$ is increasing. Then, by definition,

$$
\mathbf{K}=A(\varnothing)^{-1} A^{e x t}(\varnothing)
$$

satisfies the relation (A.1).

This way, we see that we can build a matrix $\mathbf{C}(\lambda)$ that satisfies (5.7): simply define

$$
\mathbf{C}(\lambda)=\mathbf{K C}^{e x t}(\lambda)
$$

where $\mathbf{C}^{\text {ext }}(\lambda)$ is an explicit matrix which encodes the decomposition of $s_{\lambda}(u)$ into monomials; for example in the case of $\mathbf{C}_{z}^{e x t}:=\sum_{i=0}^{n-1} \mathbf{C}^{e x t}\left(e_{i}\right) z^{i}$ corresponding to multiplication by $\prod_{i}\left(1+z u_{i}\right)$, we get

$$
\mathbf{C}_{z}^{\text {ext } \beta}{ }_{\alpha}= \begin{cases}z^{\sum_{i}\left(\alpha_{i}-\beta_{i}\right)} & \text { if } \alpha_{i}-\beta_{i} \in\{0,1\} \text { for all } i \\ 0 & \text { otherwise }\end{cases}
$$

The same procedure works of course for $\tilde{\mathbf{C}}(\lambda)$.

This construction of $\mathbf{C}(\lambda), \tilde{\mathbf{C}}(\lambda)$ is not very explicit because it assumes the knowledge of $\mathbf{K}$, which requires to compute $A^{\text {ext }}(\varnothing)$ and to invert $A(\varnothing)$. We provide a simpler formula in the next section. 


\section{B The matrices $\mathbf{P}, \mathbf{P}^{e x t}$}

We describe the change of basis from increasing sequences to link patterns, given by the matrix $\mathbf{P}$. In fact we describe a slightly bigger matrix, $\mathbf{P}^{\text {ext }}$, which allows for more general (non-decreasing) sequences. This is borrowed from appendix A of [10] and since these results are not needed in the rest of the paper, we do not provide their proof and refer to [10] for details.

Define $U_{i}:=1,-1,0$ depending on whether $i=0,1,2 \bmod 3$ (these are evaluations of Chebyshev polynomials at the parameter $\boldsymbol{\tau}=1)$. Given a sequence of integers $\alpha=\left(\alpha_{i}\right)$, define

$$
\mathbf{P}^{\pi}{ }_{\alpha}=\prod_{\substack{0 \leqslant i<j<2 n \\ i \text { and } j \text { paired by } \pi}} U_{\#\left\{\ell: i \leqslant \alpha_{\ell}<j\right\}-(j-i+1) / 2}
$$

Let us call $\mathbf{P}$ the matrix of $\mathbf{P}_{\alpha}^{\pi}$ in which $\alpha \in \mathbf{A}_{n}$, and $\mathbf{P}^{e x t}$ to be given by

$$
\mathbf{P}^{e x t}=\mathbf{P K}
$$

that is the matrix of passage from any sequence $\alpha$ to link patterns $\pi$ (see appendix A). The claim is that (B.1) provides the entries of $\mathbf{P}^{\text {ext }}$ for any non-decreasing sequence $\alpha$.

As an application, let us provide a direct way of computing $\mathbf{c}(\lambda):=\mathbf{P C}(\lambda) \mathbf{P}^{-1}$, the matrix that implements multiplication by $s_{\lambda}(u)$ in the link pattern basis. Using (A.4) and (B.2), we find

$$
\mathbf{c}(\lambda):=\mathbf{P C}(\lambda) \mathbf{P}^{-1}=\mathbf{P}^{e x t} \mathbf{C}^{e x t} \mathbf{P}^{-1}
$$

Consider now the generating function of elementary symmetric functions corresponding to multiplying by $\prod_{i}\left(1+z u_{i}\right)$ :

$$
\mathbf{c}_{z}:=\sum_{i=0}^{n-1} \mathbf{c}\left(e_{i}\right) z^{i}
$$

It is easy to check that starting from a monomial $\prod_{i} u_{i}^{-\alpha_{i}}$ with an increasing sequence $\alpha$ and multiplying by $\prod_{i}\left(1+z u_{i}\right)$, one produces sums of monomials with non-decreasing sequences of powers. Thus one can use (B.1) (and (A.5)) to calculate explicitly $\mathbf{c}_{z}$ using (B.3). Any $\mathbf{c}(\lambda)$ can then be computed by applying say the Von Nägelsbach-Kostka identity (dual Jacobi-Trudi identity). If one is only interested in $\mathbf{c}=\mathbf{c}_{1}$, the matrix that appears in recurrence formulae (cf. (5.29)) one can of course directly set $z=1$.

\section{Example of ground state entry of the Temperley- Lieb loop model}

We consider the model in size $2 n=6$. The basis is ordered as follows: $\varnothing, \square, \square, \square$, $\Gamma_{n}=\square$ (modulo the bijection to link patterns of Section 2.1). The matrices of the $\mathbf{e}_{i}$ 
are:

$$
\begin{aligned}
& \mathbf{e}_{1}=\left(\begin{array}{lllll}
0 & 0 & 0 & 0 & 0 \\
0 & 0 & 0 & 0 & 0 \\
0 & 1 & 1 & 0 & 0 \\
0 & 0 & 0 & 0 & 0 \\
1 & 0 & 0 & 1 & 1
\end{array}\right) \\
& \mathbf{e}_{2}=\left(\begin{array}{lllll}
0 & 0 & 0 & 0 & 0 \\
1 & 1 & 1 & 0 & 0 \\
0 & 0 & 0 & 0 & 0 \\
0 & 0 & 0 & 1 & 1 \\
0 & 0 & 0 & 0 & 0
\end{array}\right) \\
& \mathbf{e}_{3}=\left(\begin{array}{lllll}
1 & 1 & 0 & 0 & 0 \\
0 & 0 & 0 & 0 & 0 \\
0 & 0 & 0 & 0 & 0 \\
0 & 0 & 0 & 0 & 0 \\
0 & 0 & 1 & 1 & 1
\end{array}\right) \\
& \mathbf{e}_{4}=\left(\begin{array}{lllll}
0 & 0 & 0 & 0 & 0 \\
1 & 1 & 0 & 1 & 0 \\
0 & 0 & 1 & 0 & 1 \\
0 & 0 & 0 & 0 & 0 \\
0 & 0 & 0 & 0 & 0
\end{array}\right) \\
& \mathbf{e}_{5}=\left(\begin{array}{lllll}
0 & 0 & 0 & 0 & 0 \\
0 & 0 & 0 & 0 & 0 \\
0 & 0 & 0 & 0 & 0 \\
0 & 1 & 0 & 1 & 0 \\
1 & 0 & 1 & 0 & 1
\end{array}\right) \\
& \mathbf{e}_{6}=\left(\begin{array}{lllll}
1 & 0 & 0 & 0 & 1 \\
0 & 1 & 1 & 1 & 0 \\
0 & 0 & 0 & 0 & 0 \\
0 & 0 & 0 & 0 & 0 \\
0 & 0 & 0 & 0 & 0
\end{array}\right)
\end{aligned}
$$

so that

$$
\mathbf{H}=\left(\begin{array}{lllll}
2 & 1 & 0 & 0 & 1 \\
2 & 3 & 2 & 2 & 0 \\
0 & 1 & 2 & 0 & 1 \\
0 & 1 & 0 & 2 & 1 \\
2 & 0 & 2 & 2 & 3
\end{array}\right)
$$

with Perron-Frobenius eigenvector

$$
\psi^{\prime}=\left(\begin{array}{lllll}
1 & 2 & 1 & 1 & 2
\end{array}\right)
$$

\section{Example of matrices $\bar{A}$ and C}

We again provide data for $n=3$. The basis is ordered as in the previous section, but in this section the matrices are as defined in Sections 4 and 5 i.e. we do not use the link pattern basis. To recover the data of [22] or of the previous section, one needs to use the change of basis (cf. (2.5)) given by the matrix

$$
\mathbf{P}=\left(\begin{array}{lllll}
1 & 0 & 1 & 0 & 0 \\
0 & 1 & 0 & 0 & 0 \\
0 & 0 & 1 & 0 & 0 \\
0 & 0 & 0 & 1 & 0 \\
0 & 0 & 0 & 0 & 1
\end{array}\right)
$$


First we give the $A_{\sigma, \alpha, \tau}$ under the form of the matrices $\bar{A}$ :

$$
\begin{gathered}
\bar{A}(\varnothing)=\left(\begin{array}{ccccc}
1 & 0 & 0 & 0 & 0 \\
0 & 0 & 0 & 0 & 0 \\
0 & 0 & 0 & 0 & 0 \\
0 & 0 & 0 & 0 & 0 \\
0 & 0 & 0 & 0 & 0
\end{array}\right) \\
\bar{A}(\boxminus)=\left(\begin{array}{ccccc}
7 & 3 & 1 & 0 & 0 \\
4 & 1 & 0 & 0 & 0 \\
0 & 0 & 0 & 0 & 0 \\
1 & 0 & 0 & 0 & 0 \\
0 & 0 & 0 & 0 & 0
\end{array}\right) \\
\bar{A}(\square)=\left(\begin{array}{ccccc}
17 & 13 & 4 & 3 & 1 \\
13 & 7 & 1 & 1 & 0 \\
4 & 1 & 0 & 0 & 0 \\
3 & 1 & 0 & 0 & 0 \\
1 & 0 & 0 & 0 & 0
\end{array}\right)
\end{gathered}
$$

Summing these objects, cf. (4.7), reproduces $\Psi$ :

$$
\Psi=\left(\begin{array}{lllll}
1 & 2 & 2 & 1 & 2
\end{array}\right)
$$

Note that one possible choice in (4.7) is to set $k=0$; since $P_{\lambda}(0)=\delta_{\lambda}^{\varnothing}$, one sums in this case the first lines of the matrices $\bar{A}$ only, with coefficients $P_{\tau^{\prime}}(-n+1)$ which is nothing but $(-1)^{|\tau|}$ times the dimension of $\tau$ viewed as a $G L(n-1)$ representation. In the present case, we find $1,-2,1,3,-2$. Of course the same linear combination works when summing the first rows only ( set $k=n-1$ ).

Next, let us describe the various matrices of the representations of $\Lambda_{n}$. The $\mathcal{C}$ are Littlewood-Richardson coefficients, so they are known. Using the matrix

$$
A(\varnothing)=\left(\begin{array}{ccccc}
1 & 4 & 7 & 6 & 17 \\
0 & 1 & 3 & 4 & 13 \\
0 & 0 & 1 & 0 & 4 \\
0 & 0 & 0 & 1 & 3 \\
0 & 0 & 0 & 0 & 1
\end{array}\right)
$$


one can build the $\mathbf{C}$ :

$$
\begin{aligned}
& \mathcal{C}(\varnothing)=\left(\begin{array}{ccccc}
1 & 0 & 0 & 0 & 0 \\
0 & 1 & 0 & 0 & 0 \\
0 & 0 & 1 & 0 & 0 \\
0 & 0 & 0 & 1 & 0 \\
0 & 0 & 0 & 0 & 1
\end{array}\right) \quad \mathbf{C}(\varnothing)=\left(\begin{array}{ccccc}
1 & 0 & 0 & 0 & 0 \\
0 & 1 & 0 & 0 & 0 \\
0 & 0 & 1 & 0 & 0 \\
0 & 0 & 0 & 1 & 0 \\
0 & 0 & 0 & 0 & 1
\end{array}\right) \\
& \mathcal{C}(\square)=\left(\begin{array}{ccccc}
0 & 0 & 0 & 0 & 0 \\
1 & 0 & 0 & 0 & 0 \\
0 & 1 & 0 & 0 & 0 \\
0 & 1 & 0 & 0 & 0 \\
0 & 0 & 1 & 1 & 0
\end{array}\right) \quad \mathbf{C}(\square)=\left(\begin{array}{ccccc}
0 & 1 & -1 & 0 & 0 \\
0 & 0 & 1 & 1 & 0 \\
0 & 0 & 0 & 0 & 1 \\
0 & 0 & 0 & 0 & 1 \\
0 & 0 & 0 & 0 & 0
\end{array}\right) \\
& \mathcal{C}(\boxminus)=\left(\begin{array}{ccccc}
0 & 0 & 0 & 0 & 0 \\
0 & 0 & 0 & 0 & 0 \\
1 & 0 & 0 & 0 & 0 \\
0 & 0 & 0 & 0 & 0 \\
0 & 1 & 0 & 0 & 0
\end{array}\right) \quad \mathrm{C}(\boxminus)=\left(\begin{array}{ccccc}
0 & 0 & 1 & 0 & 0 \\
0 & 0 & 0 & 0 & 1 \\
0 & 0 & 0 & 0 & 0 \\
0 & 0 & 0 & 0 & 0 \\
0 & 0 & 0 & 0 & 0
\end{array}\right) \\
& \mathcal{C}(\square)=\left(\begin{array}{ccccc}
0 & 0 & 0 & 0 & 0 \\
0 & 0 & 0 & 0 & 0 \\
0 & 0 & 0 & 0 & 0 \\
1 & 0 & 0 & 0 & 0 \\
0 & 1 & 0 & 0 & 0
\end{array}\right) \quad \mathbf{C}(\square)=\left(\begin{array}{ccccc}
0 & 0 & 0 & 1 & -1 \\
0 & 0 & 0 & 0 & 1 \\
0 & 0 & 0 & 0 & 0 \\
0 & 0 & 0 & 0 & 0 \\
0 & 0 & 0 & 0 & 0
\end{array}\right) \\
& \mathcal{C}(\square)=\left(\begin{array}{ccccc}
0 & 0 & 0 & 0 & 0 \\
0 & 0 & 0 & 0 & 0 \\
0 & 0 & 0 & 0 & 0 \\
0 & 0 & 0 & 0 & 0 \\
1 & 0 & 0 & 0 & 0
\end{array}\right) \quad \mathrm{C}(\square)=\left(\begin{array}{ccccc}
0 & 0 & 0 & 0 & 1 \\
0 & 0 & 0 & 0 & 0 \\
0 & 0 & 0 & 0 & 0 \\
0 & 0 & 0 & 0 & 0 \\
0 & 0 & 0 & 0 & 0
\end{array}\right)
\end{aligned}
$$

so that finally,

$$
\mathbf{C}=\mathbf{C}(\varnothing)+\mathbf{C}(\square)+\mathbf{C}(\boxminus)=\left(\begin{array}{lllll}
1 & 1 & 0 & 0 & 0 \\
0 & 1 & 1 & 1 & 1 \\
0 & 0 & 1 & 0 & 1 \\
0 & 0 & 0 & 1 & 1 \\
0 & 0 & 0 & 0 & 1
\end{array}\right)
$$

The dual versions $\tilde{\mathcal{C}}, \tilde{\mathbf{C}}$ can be obtained by use of the involution whose matrix is

$$
\mathcal{I}=\left(\begin{array}{ccccc}
1 & 0 & 0 & 0 & 0 \\
0 & 1 & 0 & 0 & 0 \\
0 & 1 & 0 & 1 & 0 \\
0 & -1 & 1 & 0 & 0 \\
0 & -1 & 1 & -1 & 1
\end{array}\right)
$$

and will not be listed here. 


\section{References}

[1] M. Batchelor, J. de Gier, and B. Nienhuis, The quantum symmetric XXZ chain at $\Delta=-1 / 2$, alternating-sign matrices and plane partitions, J. Phys. A 34 (2001), no. 19, L265-L270, arXiv: cond-mat/0101385. MR

[2] L. Cantini and A. Sportiello, Proof of the Razumov-Stroganov conjecture, 2010, arXiv:1003.3376.

[3] F. Caselli and C. Krattenthaler, Proof of two conjectures of Zuber on fully packed loop configurations, J. Combin. Theory Ser. A 108 (2004), no. 1, 123-146, arXiv:math/0312217. MR

[4] F. Caselli, C. Krattenthaler, B. Lass, and P. Nadeau, On the number of fully packed loop configurations with a fixed associated matching, Electron. J. Combin. 11 (2004/06), no. 2, Research Paper 16, 43 pp, arXiv:math/0502392. MR

[5] J. de Gier, Loops, matchings and alternating-sign matrices, Discrete Math. 298 (2005), no. 1-3, 365-388, arXiv:math/0211285. MR

[6] P. Di Francesco, Totally symmetric self-complementary plane partitions and the quantum Knizhnik-Zamolodchikov equation: a conjecture, J. Stat. Mech. Theory Exp. (2006), no. 9, P09008, 14 pp, arXiv: cond-mat/0607499. MR

[7] P. Di Francesco and P. Zinn-Justin, Around the Razumov-Stroganov conjecture: proof of a multi-parameter sum rule, Electron. J. Combin. 12 (2005), Research Paper 6, 27 pp, arXiv:math-ph/0410061. MR

[8] _, Quantum Knizhnik-Zamolodchikov equation, generalized RazumovStroganov sum rules and extended Joseph polynomials, J. Phys. A 38 (2005), no. 48, L815-L822, arXiv:math-ph/0508059, doi. MR

[9] _ Quantum Knizhnik-Zamolodchikov equation: reflecting boundary conditions and combinatorics, J. Stat. Mech. Theory Exp. (2007), no. 12, P12009, 30 pp, arXiv:0709.3410, doi. MR

[10] — Quantum Knizhnik-Zamolodchikov equation, totally symmetric selfcomplementary plane partitions and alternating sign matrices, Theor. Math. Phys. 154 (2008), no. 3, 331-348, arXiv:math-ph/0703015, doi.

[11] P. Di Francesco, P. Zinn-Justin, and J.-B. Zuber, A bijection between classes of fully packed loops and plane partitions, Electron. J. Combin. 11 (2004), no. 1, Research Paper 64, 11 pp, arXiv:math/0311220. MR

[12] _ Determinant formulae for some tiling problems and application to fully packed loops, Ann. Inst. Fourier (Grenoble) 55 (2005), no. 6, 2025-2050, arXiv:math-ph/0410002. MR

[13] T. Fonseca and P. Zinn-Justin, On the doubly refined enumeration of alternating sign matrices and totally symmetric self-complementary plane partitions, Electron. J. Combin. 15 (2008), Research Paper 81, 35 pp, arXiv:0803.1595. MR 
[14] R. Langer, Symmetric functions and Macdonald polynomials, Lambert Academic Publishing, 2010, Master's thesis.

[15] P. Nadeau, Fully packed loop configurations in a triangle I, work in progress.

[16] _ Fully packed loop configurations in a triangle II, work in progress.

[17] V. Pasquier, Quantum incompressibility and Razumov Stroganov type conjectures, Ann. Henri Poincaré 7 (2006), no. 3, 397-421, arXiv: cond-mat/0506075. MR

[18] J. Propp, The many faces of alternating-sign matrices, Discrete models: combinatorics, computation, and geometry (Paris, 2001), Discrete Math. Theor. Comput. Sci. Proc., AA, Maison Inform. Math. Discrèt. (MIMD), Paris, 2001, pp. 043-058, arXiv:math/0208125. MR

[19] A. Razumov and Yu. Stroganov, Combinatorial nature of the ground-state vector of the O(1) loop model, Teoret. Mat. Fiz. 138 (2004), no. 3, 395-400, arXiv:math/0104216, doi. MR

[20] A. Razumov, Yu. Stroganov, and P. Zinn-Justin, Polynomial solutions of $q K Z$ equation and ground state of $X X Z$ spin chain at $\Delta=-1 / 2$, J. Phys. A 40 (2007), no. 39, 11827-11847, arXiv:0704.3542, doi. MR

[21] K. Shigechi and P. Zinn-Justin, Path representation of maximal parabolic KazhdanLusztig polynomials, 2010, preprint, arXiv:1001.1080.

[22] J. Thapper, Refined counting of fully packed loop configurations, Sém. Lothar. Combin. 56 (2006/07), B56e, 27 pp, http://www.emis.de/journals/SLC/wpapers/s56thapper.html. MR

[23] B. Wieland, A large dihedral symmetry of the set of alternating sign matrices, Electron. J. Combin. 7 (2000), Research Paper 37, 13 pp, arXiv:math/0006234. MR

[24] D. Zeilberger, Proof of a conjecture of Philippe Di Francesco and Paul Zinn-Justin related to the $q K Z$ equations and to Dave Robbins' two favorite combinatorial objects, 2007 http: //www . math. rutgers. edu/ zeilberg/mamarim/mamarimhtml/diFrancesco.html.

[25] J.-B. Zuber, On the counting of fully packed loop configurations: some new conjectures, Electron. J. Combin. 11 (2004), no. 1, Research Paper 13, 15 pp, arXiv:math-ph/0309057. MR 